\title{
Is the new "energetic feedback" for insulin delivery monitoring available in any acute severe glucose metabolism disorders?
}

\begin{abstract}
Background: Taking into account the remaining difficulties of managing insulin delivery without hypoglycaemia and yo-yo events, a new feedback based on differential temperature evaluation $(\Delta \mathrm{T})$ was previously proposed and successfully tested with a device named ADD-CIT (apparatus-for-diabetes-diagnosis-complex-forinsulin-therapy) on acute decompensated Diabetes 1 patients. The present work aim is to verify whether ADD-CIGT can be useful for insulin delivery management in any acute severe hyperglycaemia independently on its origin.
\end{abstract}

Material and methods: Three groups of patients were considered: DM-1 (83), DM-2 (45) and without known diabetes DM-0 (66). In each group part of patients was treated as usually (UT), part-by ADD-CIT. Healthy volunteers (15) underwent body temperature observation for determination of its normal evolution. Glycaemia was measured once/hour (gasometer or prickle method). Differential body temperatures were registered by the device.

Results: In the ADD-CIT subgroups a $30-40 \%$ glycaemia decrease from its initial value was observed within 2-4 hours versus 5-7 hours in the UT subgroups. Episodes of hypoglycaemia $(<4.3 \mathrm{mMole} / \mathrm{l})$ were registered in $15 \%$ of the ADD-CIT sessions (10 patients, all DM1), versus $22.5 \%$ in the UT subgroups. Yo-yo phenomena were exceptional in ADD-CIT subgroups and frequent in UT. $\Delta \mathrm{T}$ reactions occurred about 1 hour before glycaemia ones that might explain the better adaptation of insulin delivery to the patient's needs in ADD-CIT subgroups than in UT ones. $\Delta \mathrm{T}$ levels approaching values observed in healthy persons signed stopping the session. Sometimes $\Delta \mathrm{T}$ decreased at the session end, glycaemia remaining stable: then a cautious glucose injection corrected the situation without glycaemia modification.

Conclusion: The ADD-CIT patients have shown faster glycaemia decrease and stabilization, less hypoglycaemia events than UT patients independently on hyperglycaemia causes. This supports the hypothesis that $\Delta \mathrm{T}$ evolution registration is useful as a complementary feedback for insulin delivery management in any patients with acute severe hyperglycaemia.

Keywords: diabetes mellitus, insulin therapy, glucose metabolism disorders, energetic balance, artificial pancreas
Volume 6 Issue 5 - 2018

\author{
VK Novikov, ${ }^{1,3}$ SA Dobos, ${ }^{2}$ J Devriendt, ${ }^{2}$ V \\ Coulic' \\ 'Translational Research Laboratory, ULB, Belgium \\ ${ }^{2} \mathrm{CHU}$ Brugmann (Departments of Intensive Care, of Abdominal, \\ Thoracic and Laparoscopic Surgery), Belgium \\ ${ }^{3} \mathrm{VN}$ Sklifosovski Scientific Research Institute of Emergency \\ Medicine, Russia
}

Correspondence: Coulic Very, Translational Research Laboratory, ULB; Brussels, Belgium, 2 avenues JJ Crocq, 1020 Brussels, Belgium, Tel 322477 2574, Email coulac.very@belgacom.net

Received: July 30, 2018| Published: October 22, 2018
Abbreviations: ADD-CIT, apparatus for diabetes diagnosis and complex for insulin therapy; ARCA, Acute cardio-respiratory arrest; BW, body weight; DM1, type 1 diabetes mellitus; DM2, type 2 diabetes mellitus; DM0, non-diabetic patients; $\Delta \mathrm{T}$, difference between core and superficial temperatures; HD, heart disease; ICU, Intensive Care Unit; Tc, core temperature; Ts, superficial temperature; UT, Usual treatment

\section{Introduction}

Up to now glycaemia measure remains the only criterion used for the treatment management of hyperglycaemia in diabetic or critically ill (ARDS, shock) patients and in spite of interesting innovations including glucagon administration, artificial pancreas, close loop use and others, hypoglycaemia episodes and yo-yo events are still a problem during insulin therapy. ${ }^{1-6}$ According to some authors ${ }^{7-9}$ and our previous observations, glycaemia reaction is too slow to reflect the real metabolism situation. Hence the correction is started a little late, excessive doses administration and answers to them cannot be avoided, even if mathematic anticipation is provided, and that cause the aforementioned events. ${ }^{7-9}$

Besides, glycaemia is known to be the result of the difference between glucose production and utilization which depend on many changing factors, such as external glucose intake, internal hormonal influences, neo gluconeogenesis, physical and intellectual activities. Taking into account that $70 \%$ of the energy used by the organism comes from glucose turn over ${ }^{10}$ it was likely that the basal metabolism evolution could better reflect the organism needs in insulin and glucose than glycaemia measure, allowing anticipation of its fluctuations. But presently, direct basal metabolism measure is no more performed and not even feasible in current clinics. Indirect representation of the energetic balance evolution, based on the dynamics of the difference between the body core temperature and the temperature of the body superficial layers, has seemed possible.

Some years ago an alternative feedback for insulin delivery monitoring was proposed. ${ }^{11-12}$ 
The hypothesis was that continuous record of $\Delta \mathrm{T}$, (differential temperature $\Delta \mathrm{T}=$ core temperature $\mathrm{Tc}$-superficial temperature $\mathrm{Ts}$ ) represents the result of energy production and utilization, thus reflects glucose metabolism, and hence can be a valuable complement to blood glucose measure for monitoring insulin therapy . Devices were created and tested which used $\Delta \mathrm{T}$ evolution as a feedback for automated insulin delivery.

.Recently the Apparatus for Diabetes Diagnosis coupled with Complex for Insulin Therapy (ADD-CIT), using this kind of feedback has been shown to be useful in the treatment monitoring of acute life threatening alterations of the glucose metabolism due to type 1 diabetes. ${ }^{13-16}$ But is it able to correct glucose metabolism disorders of other origin that is in any acute situation of hyperglycaemia?

The objective of the present work is to verify the assertion that ADD-CIT could be successfully used in any severe, acute glucose metabolism disorders when insulin treatment is required, temporarily or not, in different groups of diabetic and non-diabetic patients.

\section{Material and methods}

Three groups of patients were considered: with type 1 diabetes (DM-1-83); with type 2 diabetes (DM-2-45); and patients without known diabetes (DM-0-66) but presenting a severe pathology also causing acute hyperglycaemia difficult to control. The evolution of glycaemia and $\Delta \mathrm{T}$ was also investigated in a group of 15 healthy volunteers.

Part of these patients were treated according usual schemas (UT), the other part was treated by ADD-CIT (Table 1). The main inclusion criterion was the presence of hyperglycaemia $>11.1 \mathrm{mmol} / 1$ $(200 \mathrm{mg} / \mathrm{dl})$ at admission. The request of the treating physician was also necessary. Exclusion criteria were: age $<16$, gravidity, rectal pathology. Age, sex, BW were comparable. Observation or treatment session duration was 2-5 hours as a rule, exceptionally up to 8 hours, and always provided under the supervision of an experimented ICU physician. The device and its use were previously described ${ }^{13-16}$ (Figure 1).

Table I Patient cohort

\begin{tabular}{|c|c|c|c|c|}
\hline Series & DM I & DM 2 & DM 0 & Healthy control \\
\hline $\begin{array}{l}\text { A D D - C I T } \\
\text { treated } \\
(\mathrm{N}=\mid 42)\end{array}$ & $\begin{array}{l}64 \text { (including } 50 \text { with kidney } \\
\text { insufficiency) }\end{array}$ & $\begin{array}{l}36 \text { (including } 18 \text { with heart } \\
\text { pathology) }\end{array}$ & 27 (with heart pathology) & (observation \\
\hline $\begin{array}{l}\text { Usual schema } \\
(\mathrm{N}=67)\end{array}$ & 19 & 9 & $\begin{array}{l}39 \text { (24 ARCA, } 15 \text { with } \\
\text { heart pathology) }\end{array}$ & 0 \\
\hline Total & 83 & 45 & 66 & 15 \\
\hline
\end{tabular}

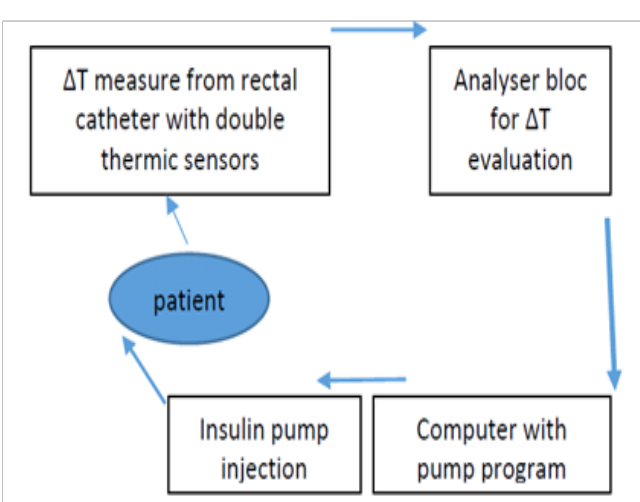

Figure I ADD-CIT design schema:

I: rectal catheter with, 2: Thermic sensors for core (Tc) and superficial (Ts) body temperature, 2.Analyser, 3: Computer for insulin pump programming from $\Delta \mathrm{T}$ evolution data, 4 : Insulin pump.

\section{For reminding 1. Description of the device}

The rectal catheter (1) enclosed into a thin, hermetic, waterproof and one-using polyethylene membrane, has a $4 \mathrm{~mm}$ diameter and $8 \mathrm{~cm}$ length. The two temperature sensors are at $4.5 \mathrm{~cm}$ from each other to avoid temperature "contamination" between them. When the catheter is introduced, the inner sensor at its top is placed at the level of the deep haemorrhoidal venous plexus and registers the core temperature (Tc) reflecting thermogenesis; the lower sensor is placed just above the external anal sphincter and reflects the superficial temperature (Ts) of the subcutaneous para-rectal fat.

The sensors data are cabled to an analyser (2) which calculates $\Delta \mathrm{T}$ and the information is delivered to a computer (3). The evolution of $\Delta \mathrm{T}$ is used for the elaboration of a program which is transmitted by the computer to an insulin pump (4) adapted in consequence. InsulinActrapid or Humulin, that is of rapid action, diluted to 20 or $40 \mathrm{UI} / \mathrm{ml}$, is delivered through a short $(<20 \mathrm{~cm})$ catheter to the patient venous access to avoid insulin adsorption and degradation. Insulin delivery might be operator programmed (30 impulses/min usually during 30 minutes at the session beginning while sensors adapt) or automatized according to $\Delta \mathrm{T}$ values evolution. 1 impulse of the pump represents from 0.0003 to $0.001 \mathrm{UI}$ according to the insulin pre dilution. The program regulates the number of impulses/min. A visual control of the $\Delta \mathrm{T}, \mathrm{Tc}$ and $\mathrm{Ts}$ ciphers, and insulin delivery impulses was ensured through the computer.

\section{Description of the procedure}

The investigations were provided in a hospital (ICU) room at temperature $2 \mathrm{I}+/-1^{\circ} \mathrm{C}$, under the permanent observation and responsibility of an intensive care physician after receiving the patient's own informed consent or his family's. The responsible physician mildly introduced the catheter with the sensors into rectum at a depth of $6.5 \mathrm{~cm}$ (mark on the catheter). An intravenous connection was placed independent on the patient injection system or connected with it for intravenous insulin providing. Usually patients did not receive any food except in the case when session duration exceeded 4 hours and the patient was conscious. In the last case standard hospital meal was delivered.

At the end of the session, rectal catheter was carefully taken out the organism and pump insulin delivering - removed. The session might be interrupted in the case of emergency procedures, defecation or disagreeable anal feelings (the last was not observed in this trial). Criteria for ending a session were objective significant decrease of blood glucose level $(<11.1 \mathrm{mM} / \mathrm{l})$ and $\Delta \mathrm{T}>0.05^{\circ} \mathrm{C}$ with subjective improvement of the patient's condition. After ADD or ADD-CIT procedure patients returned to ICU responsibility or were transferred 
into other units. Late observation was never provided by our teams.

As far as glycaemia is the universal criterion of severity and treatment of hyperglycaemia, it was used here for control of the device work. Evaluation of the capillary glycaemia was performed once an hour by strip method (One Touch, Switzerland or Acutrend, USA) and controlled each 3 hours by arterial glucometer ("ADL-90 Flex"-or "Glucose and lactate Analyser", Eco Basic United, Moskau). Usual in ICU clinical investigations were provided.

Glycaemia was considered as normal if included between 3.88 and $7.7 \mathrm{mM} / 1$ (70 and $140 \mathrm{mg} / \mathrm{dl})$, increased but acceptable between 7.71 and $11.1 \mathrm{mM} / \mathrm{dl}$ (141 and $200 \mathrm{mg} / \mathrm{dl}$ ), moderately high between 11.11 and $15.0(200$ and $270 \mathrm{mg} / \mathrm{l})$; high between 15.1 and $20.0 \mathrm{mM} / 1(270$ and $360 \mathrm{mg} / \mathrm{dl}$ ); very high between 20.1 and 25.0 (360 and $450 \mathrm{mg} /$ $\mathrm{dl})$; threatening high $>25.1(>450 \mathrm{mg} / \mathrm{dl})$. Maximum observed was 33 $\mathrm{mM} / 1(599,4$, i.e. $600 \mathrm{mg} / \mathrm{dl})$.

$\Delta \mathrm{T}<0.05$ and $>0.2^{\circ} \mathrm{C}$ was considered as pathological. $\mathrm{NB} \Delta \mathrm{T}$ at hour 0 that is at the moment of introducing the rectal catheter cannot be registered because a $30 \mathrm{~min}$ time is necessary for adaptation of thermic sensors to organism conditions.

Statistic evaluation was provided using Average or Mean +/-
Standard Deviation (M+/-SD) calculation and Tc against Tst Student criterion determination of liability (p). Involvement of other not parametric statistic was considered but did not change the results. Correlation between $\Delta \mathrm{T}$ and glycaemia was not calculated considering the probable discordance in time of the two parameters..$^{7-9,16}$

Data have been collected mainly in the ICU service of the Moscow Emergency Institute named by VN. Sklifossovski, from 2001 till 2016 years with the agreement of the Russian Federation Ministry of Health. The investigation provided in the Brugmann CHU Hospital was also authorized by the local Ethics Committee.

\section{Results}

In the ADD-CIT group of patients a glycaemia decrease of 30 $40 \%$ from its initial value was observed within 2-4 hours and a stable mean glucose level equal or inferior to $11.1 \mathrm{mmole} / \mathrm{l}(200 \mathrm{mg} / \mathrm{dl})$ was obtained. (Figure 2A). 20 episodes of hypoglycaemia $(<4.3$ mmole/l) during 131 sessions that represents $15 \%$ of events which have involved 13 patients, that is $9.9 \%$ of the patients (some of them presented several episodes during one session. Interesting to note that only DM 1 patients were concerned. Hypoglycaemia episodes were absent in DM 2 and DM 0 patients treated with the help of ADD-CIT. No yo-yo phenomena were observed in any series (Table 2).

Table 2 Hypoglycaemia episodes in patients treated with ADD-CIT use or classical method

\begin{tabular}{lllllllll}
\hline Series & \multicolumn{1}{l}{ ADD-CIT (N) } & \multicolumn{3}{c}{$\begin{array}{l}\text { Classical } \\
\text { method }\end{array}$} & \multicolumn{3}{c}{$\begin{array}{l}\text { A D D - C I T I } \\
\text { CM (\%) }\end{array}$} \\
\hline & Sessions & Events & Patients & Sessions & Events & Patients & Event & Patients \\
DM I ) & 89 & 20 & 13 & 19 & 6 & 5 & $22.4 / 31.5$ & $9.9 / 26.3$ \\
DM 2 & 16 & 0 & 0 & 9 & 4 & 1 & $0 / 44$ & $0 / 11.1$ \\
DM 0 (HD) & 26 & 0 & 0 & 39 & 5 & 4 & $0 / 12.8$ & $0 / 10.25$ \\
TOTAL & I31 & 20 & 13 & 67 & 15 & 10 & $15 / 22.4$ & $9.9 / 14.9$ \\
\hline
\end{tabular}

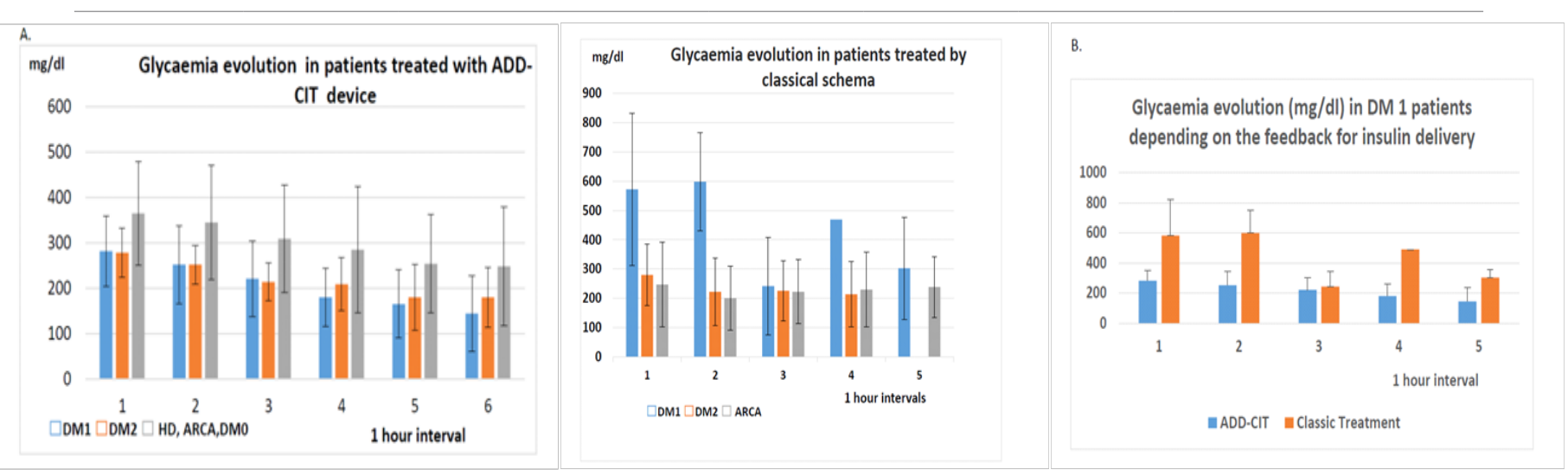
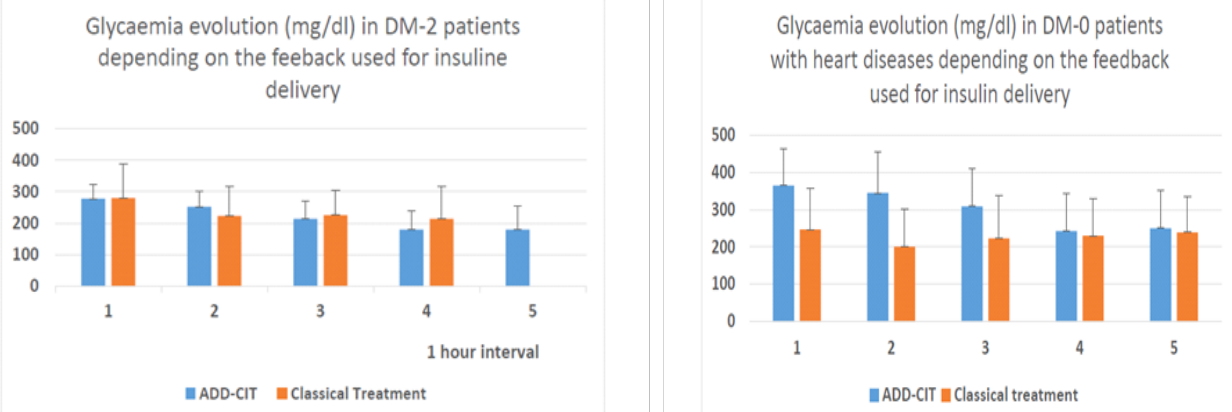

Figure 2 Graphics of glycaemia evolution in different patient groups treated with classical feedback and insulin delivery shemas versus with ADD-CIT. Series I-blue-Type I Diabetes, Series 2-red-Type 2 Diabetes, series 3-green-Heart failure in non-diabetic patients ((M \pm SD):

Comparison of ADD-CIT groups with Classical treatment groups

Evaluation of the ADD-CIT and classical treatment in each of the three pathologies represented. 
In the control groups monitored only by glycaemia evaluation, the same glycaemia decrease was attained only after 5-7 hours with frequent yo-yo phenomena even concerning mean values (Figure 2A) (Figure 2B). Significant hypoglycaemia events were also present during the 67 provided sessions: they represented $22.4 \%$ of the sessions and concerned $14.9 \%$ of the patients. The phenomena affected the 3 groups of patients.

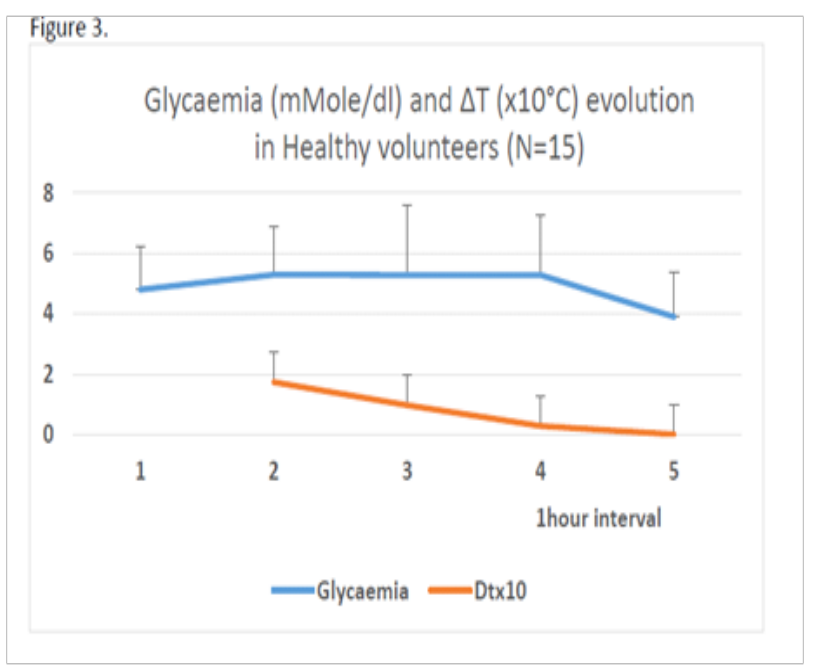

Figure 3 Evolution of glycaemia and $\Delta \mathrm{T}$ in 15 healthy patients $(M \pm S D)$ Decrease of $\Delta T$ linked with fasting appears at least I hour before glycaemia decrease.

Considering the different pathologies ADD-CIT ensured a regular mild glycaemia decrease with following stabilisation at an acceptable level (average between 140 and $200 \mathrm{mg} / \mathrm{dl}$ ) in DM 1 and DM 2 patients, whereas in HD DM0 patients the results were less demonstrative: average glycaemia remained moderately high (about $250 \mathrm{mg} / \mathrm{dl}$ ) at the end of the sessions. Classical treatment also led to a glycaemia decrease, but more chaotic with temporary re-increases especially in DM 2 and HD DM 0 patients (Figure 2). The influence of pathology on the hypoglycaemia events in terms of patients concerned and event repetition in the same patient was different and affected mainly type I diabetes patients see Table 2 .

It is to be noted that significant glycaemia average decrease could not be found because of the great dispersion of the registered values. It is the reason why the attention was concentrated on the individual percentage of the glycaemia decrease during the observation period in both subgroups.

No correlation was noted between $\Delta \mathrm{T}$ and glycaemia in hyperglycaemic patients as well as in healthy persons, though in this last group during the fourth hour of observation the values of both indices decreased (Figure 3). $\Delta \mathrm{T}$ values were negative mainly in decompensated DM 1 patients. $\Delta \mathrm{T}$ decreased during the sessions in DM 2 patients but rarely reached negative levels. At the end of the most successful sessions $\Delta \mathrm{T}$ values corresponded to values observed in healthy controls (Figure 3). This was an indication for stopping the session. In some cases $\Delta \mathrm{T}$ abnormally felt at the end of a session whereas glycaemia remained stable: at this moment a cautious glucose injection usually has corrected the situation without glycaemia modification.

The insulin amount delivered in both groups was different: a little lower in the ADD-CIT. But as far as the patients have already received insulin before the sessions in different quantities, with different efficiency, it was difficult to appreciate the significance of the differences observed because the variability of the used doses was great in both series.

\section{Discussion}

Though our series were small and not fully matched, the mean results of insulin therapy in ADD-CIT groups of patients in terms of glycaemia decrease and stabilization as well as decrease of hypoglycaemia event frequency and repeats looked convincingly better than in patients treated by the usual procedure only based on glycaemia measurements. This confirms the usefulness of $\Delta \mathrm{T}$ evolution evaluation as a complementary feedback for insulin delivery not only in DM 1 patients but also in DM 2 and any other patients with acute severe glucose metabolism disorders.

Interesting that the effect of both treatment techniques were slightly different depending on the kind of pathology concerned. ADD-CIT seemed to be more efficient in DM 1 than in DM 2 patients. On the contrary classical treatment has led to a certain stabilisation in DM 2 patient mean glycaemia though the last remained about $200 \mathrm{mg} / \mathrm{dl}$. Heart disease DM0 patients were the more difficult to be helped. That means that ADD-CIT as any other insulin treatment of hyperglycaemia has its limits: as long as the causes of the disorder are not eliminated a relapse has to be awaited. It explains the situation with ARCA patients. So ADD-CIT is only an adapted correction of acute hyperglycaemia, because $\Delta \mathrm{T}$ evolution is more sensible to metabolism variations than glycaemia: the reactions were noted within 1-2 min. instead of 30-40 min or even more. This is one of the probable explanations of the absence of correlation between glycaemia and $\Delta \mathrm{T}$.

The signification of isolated values of $\Delta \mathrm{T}$ is not clear yet, though it is known that negative values are not a good prognosis, especially if they persist. In our retrospective study we also should have to take into account 1 / the former treatment which was administrated to the patients prior to the session beginning, $2 /$ the peculiarities of the basis pathology development (with complications or not and what kind of them), their possible influence on the present status and, consequently, on initial $\Delta \mathrm{T}$. So special investigations in that direction are worthwhile to be planned.

Some other questions remain: Is $\Delta \mathrm{T}$ feedback applicable to chronic treatment of glucose metabolic disorders? To primary hypoglycaemia situations? And what may be the diagnostic or prognostic signification of $\Delta \mathrm{T}$ levels and dynamics? The first variant of our device was ADD, that means it was created with a diagnosis aim. ${ }^{11,12}$ For realization of these verifications some technical progress is to be realized, particularly the telemetric measure of Tc and Ts.

\section{Conclusion}

The ADD-CIT device based on "energetic" feedback for insulin treatment monitoring is not worse and probably more adapted to patient individual approach than classical treatment schemas, and can be proposed in any acute severe hyperglycaemia.

\section{Acknowledgments}

The authors are thankful to Professor P Bergmann (ULB Laboratory of Experimental Medicine, Brussels, Belgium) for discussing the results and to TP Pisareva ( presently retreated, Moscow) for scientific and logistic help. They have appreciated the "ideological" support of Dr P Delrée and the Institute of Pathology and Genetics (Gosselies, Belgium). 


\section{Conflict of interest}

There is no conflict of interest.

\section{References}

1. McNeilly AD, McCrimon RJ. Impaired hypoglycaemia awareness in type 1 diabetes: lessons from the lab. Diabetologia. 2018;61(4):743-750.

2. Hasanbegovic S, Obarcanin E, Hasanbegovic E, et al. Impact of insulin delivery method on hypoglycaemia incidence in pediatric type 1 diabetes mellitus patients? Med Arch. 2017;71(6):391-395.

3. Maurizi AR, Piemonte V, Pozzilli P. Diabetes on demand and novel technologies. Diabetes Metab Res Rev. 2018;34(2).

4. Namikawa T, Munekage M, Yatabe T, et al. Current status and issues of the artificial pancreas: abridged English translation of a special issue in Japanese. J Artif Organs. 2018; 21(2):132-137.

5. Blauw H, Keith-Hynes P, Koops R, et al. A review of safety and design requirements of the artificial pancreas. Ann Biomed Eng. 2016; 44(11):3158-3172

6. Laguna Sanz AJ, Mulla CM, Fowler KM, et al. Design and clinical evaluation of a new low-glucose prediction algorithm with mini-dose stable glucagon delivery in post-bariatric hypoglycaemia. Diabetes Technol Ther. 2018;20(2):127-139.

7. Harvey RA, Dassau E, Zisser H, et al. (2012) Design of the health monitoring system for the artificial pancreas: low glucose prediction module. J Diabetes Sci Technol. 2012;6(6):1345-1354.

8. Facchinetti A, Sparacino G, Guerra S, et al. Real-time improvement of continuous glucose-monitoring accuracy: the smart sensor concept. Diabetes Care. 2012;36(4):793-800
9. WilsonLM, Castle JR. Stable liquid glucagon: beyond emergency hypoglycaemia rescue. Diabetes Sci Technol. 2018; 12(4):847-853.

10. Schmidt RF, Tes G. Human Physiology. Moscow edn, Nauka, 1986.

11. Novikov VK, Coulic VP. Méthode/Procédé de diagnostic du diabète sucré"(Method of diabetes mellitus diagnosis) (priority 1987) Moscow 15.11.1991 Patent $n^{\circ} 1718822$

12. Simonov VA, Kluchko AV, Novikov VK. Electronic device for control of the energetic balance in man and animals (appliances in Diabetes Mellitus). Proceedings of the 14th Annual International Conference of the IEEE Engineering in Medicine and Biology Society, Paris, France, 1992; pp. 2270-2271.

13. Coulic V, Novikov Vi, Devriendt J. Use of temperature gradient measuring device in monitoring of diabetic and critically ill patients" in "Sciences and Technologies for Health" p.372, abstract FrP2C1.3 (29th Annual International Conference of the IEEE in conjunction with the Biennial Conference of the SFGBM, France, Lyon, 2007.

14. Coulic V, Novikov V, Devriendt J. A new feedback for monitoring insulin therapy? (First experimental and clinical tests). J Pancreatic Disorders and Therapy. 2015; S5: 005.

15. Novikov V, Anissimov Iu, Dmitriev I. Differential temperature evolution for insulin delivery monitoring in type 1 diabetic patients before and after kidney-pancreas transplantation. Int $J$ Diabetes Clin Diagn. 2017;4(3):123

16. Novikov VK, Dobos SA, Devriendt J. Energetic balance feedback for insulin delivery monitoring applied to hyperglycaemic non diabetic type I patients. WC $\Delta \mathrm{T} 2017$. 